\title{
Revealing 'plasmaron' feature in DySb by optical spectroscopy study
}

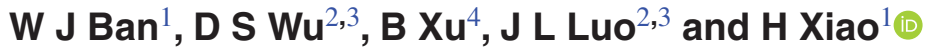 \\ ${ }^{1}$ Center for High Pressure Science and Technology Advanced Research, Beijing 100094, \\ People's Republic of China \\ 2 Beijing National Laboratory for Condensed Matter Physics, Institute of Physics, Chinese Academy of \\ Sciences, PO Box 603, Beijing 100190, People's Republic of China \\ ${ }^{3}$ School of Physical Sciences, University of Chinese Academy of Sciences, Beijing 100049, \\ People's Republic of China \\ 4 Department of Physics and Fribourg Center for Nanomaterials, University of Fribourg, \\ Chemin du Musée 3, CH-1700 Fribourg, Switzerland \\ E-mail: hong.xiao@hpstar.ac.cn
}

\begin{abstract}
We report magnetic susceptibility, resistivity and optical spectroscopy study on single crystal sample DySb. It exhibits extremely large magnetoresistance (XMR), and a magnetic phase transition from paramagnetic (PM) to antiferromagnetic (AFM) state at about $10 \mathrm{~K}$. A 'screened' plasma edge at about $4000 \mathrm{~cm}^{-1}$ is revealed by optical measurement, which suggests that the material has a low carrier density. With decreasing temperature, the 'screened' plasma edge shows a blue shift, possibly due to a decrease of the effective mass of carriers. Notably, an anomalous temperature dependent midinfrared absorption feature is observed in the vicinity of the 'screened' plasma edge. In addition, it can be connected to the inflection point in the real part of the dielectric function $\epsilon_{1}(\omega)$, the frequency of which exactly tracks the temperature dependent 'screened' plasma frequency. This phenomena can be explained by the appearance of a coupled electron-plasmon 'plasmaron' feature.
\end{abstract}

Keywords: optical conductivity, magnetoresistivity, plasmaron

\section{Introduction}

Rare-earth $(R)$ mono antimonides $R \mathrm{Sb}$ with simple NaCl-type structure are excellent candidate for both experimental and theoretical study. The rare-earths have different occupation numbers for the inner $4 f$ shell, giving rise to rich magnetic and electronic properties [1-28]. In terms of electronic structure, most $R \mathrm{Sb}$ compounds are known as compensated semimetals, which consists of two hole FS (Fermi surface) pockets at the Brillouin zone (BZ) center $\Gamma$ and one electron FS pocket at the BZ boundary $X$, with the conduction band mainly deriving from rare-earth $5 d$ states and the valence band deriving from pnictogen $5 p$ states, respectively [14-18, 20-27].

$R \mathrm{Sb}$ exhibits extremely large magnetoresistance (XMR), thus have potential applications such as spintronics devices, magnetic memory, and magnetic field sensors [14-20, 22,
25-27, 29]. They also share similar magnetotransport property with topological nontrivial semimetal, such as $\mathrm{WTe}_{2}$ [30], $\mathrm{Cd}_{3} \mathrm{As}_{2}$ [31, 32], TaAs [33], and NbP [34]. Therefore they are possible host of topologically nontrivial phases. Recently there are evidences for the existence of Dirac semimetal nodes or topological insulating gaps along $\Gamma-X$ appears in LaSb $[15,17,24]$; unusual fourfold degenerate Dirac surface state in CeSb $[20,25,26]$, and a Dirac-like structure at the $\Gamma$ point in $\mathrm{YSb}, \mathrm{NdSb}$, and $\mathrm{GdSb}[18,20]$. The property of $R \mathrm{Sb}$ is further enriched by the report of antiferromagnetic (AFM) phase transition in CeSb, NdSb, SmSb, GdSb, TbSb, DySb, HoSb, and ErSb at low temperature [18, 20-22, 28].

Optical spectroscopy is a bulk-sensitive technique with high-energy resolution, which provide useful information about charge dynamics, carrier density and band structure of a material over a broad range of energy scales. Previous 
optical spectroscopy study on $R \mathrm{Sb}(R=\mathrm{La}, \mathrm{Ce}, \mathrm{Pr}$, and $\mathrm{Sm})$ show absorptions due to the $p-d$ transition and additional ones whose intensity is proportional to the number of occupied $4 f$ electrons [35]. The optical spectrum of CeSb in its magnetic ordered state is significantly affected by the $\mathrm{Sb}$ $5 p$-Ce $4 f$ mixing effect [36]. In addition, absorptions due to a Kondo peak and which is related to virtual $f-d$ excitation are observed in far infrared and infrared regions, respectively [37]. A hump at about $0.25 \mathrm{eV}$ is observed in PrSb, GdSb and DySb, which is attributed to the intraband transition induced by the scattering between the spin of carriers and the localized magnetic moments at each site of rare-earth ion [38].

In this paper, we report magnetic susceptibility, resistivity and optical spectroscopy study on DySb, an isostructural compound of LaSb but with the presence of $4 f$ electrons. Magnetic susceptibility shows a phase transition from paramagnetic (PM) to AFM state at about $10 \mathrm{~K}$. Magnetoresistivity measurements found that DySb exhibits large magnetroresistance at low temperatures. The optical spectroscopy study shows an increase of the plasma edge, i.e. and the 'screened' plasma frequency $\left(\omega_{p}^{*}\right)$, with decreasing temperature, which is similar to LaSb [39]. In contrast to LaSb, an anomalous midinfrared absorption in $R(\omega)$ is observed in DySb. In addition, the real part of the dielectric function $\epsilon_{1}(\omega)$ has an inflection point, which is coincident with the temperature dependent 'screened' plasma frequency. This phenomenon can be explained by the appearance of the coupled electron-plasmon, that is 'plasmaron' feature, probably due to the effect of $4 f$ electrons in DySb.

\section{Experimental details}

The DySb single crystals were grown by the flux method, similar to the synthesis of LaSb [15]. Large pieces of single crystals with shiny surfaces were obtained. The resulting crystals have dimensions of several millimeters. Room-temperature $\mathrm{X}$-ray diffraction (XRD) measurements were performed on a PANalytical Empyrean diffractometer using $\mathrm{Cu} \mathrm{K} \alpha$ radiation $(\lambda=1.5418 \AA)$ in order to check the phase purity. The $d c$ resistivity measurement was conducted on a commercial quantum design physical properties measurement system (PPMS) by a four-probe method with the electrical current parallel to the $a b$ plane of the crystal. The magnetic susceptibility $\chi(T)$ was measured by using the VSM option of the PPMS system. The temperature-dependent optical reflectance data were measured via a near-normal angle of incidence on Bruker $113 \mathrm{v}$ and Vertex $80 \mathrm{v}$, on as-grown shinny surface of the single crystal from 120 to $20000 \mathrm{~cm}^{-1}$. We obtain the reflectivity $R(\omega)$ by calibrating the signal against a reference gold/aluminum layer evaporated in situ on the sample surface and then get the real part of the optical conductivity $\sigma_{1}(\omega)$ by the Kramers-Kronig analysis of $R(\omega)$. For the extrapolation at low frequency, we used the Hagen-Rubens relation ( $R=1-A \sqrt{\omega}$ ). For the extrapolation on the high frequency side, we employed an extrapolation method with $\mathrm{x}$-ray atomic scattering functions [40].

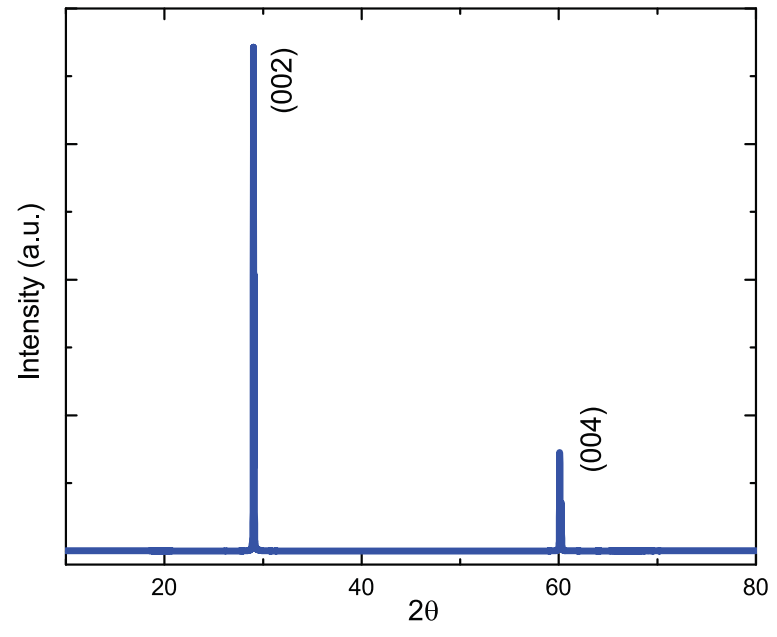

Figure 1. XRD pattern on the $(00 l)$ surface of single crystal DySb.

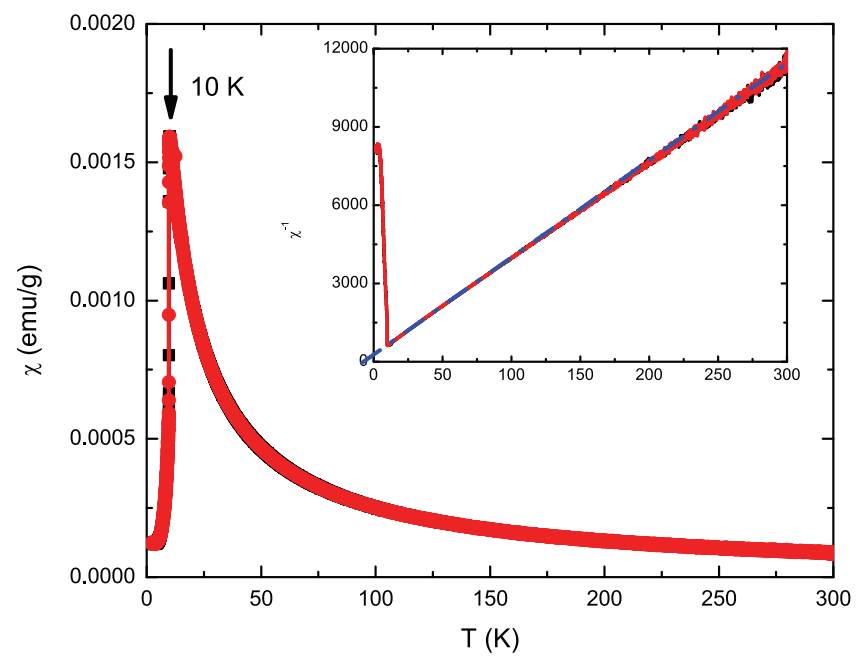

Figure 2. The temperature dependent magnetic susceptibility $[\chi=M(T) / H]$ of DySb measured under ZFC and FC conditions. The black arrow indicate the phase transition from paramagnetic to AFM state. Inset: the reciprocal magnetic susceptibility $1 / \chi$ versus $T$ of DySb.

\section{Results and discussions}

Figure 1 shows the XRD patterns for the samples of DySb, in which only $(00 l)$ peaks are observed, confirming high quality of the single crystal, which is consistent with previous reports [41].

Figure 2 shows the temperature dependence of the magnetic susceptibility $\chi$ of DySb under zero-field-cooled (ZFC) and field-cooled (FC) conditions, measured in a magnetic field of $0.1 \mathrm{~T}$ applied along the $a b$ plane. There is no evident difference between the ZFC and FC curves, which is a common feature in paramagnetic and AFM state. At high temperatures, the $\chi(T)$ curve can be well fitted by the Curie-Weiss law (inset to figure 2) adopting the Curie Weiss temperature $\Theta=-7.8 \mathrm{~K}$ and the effective magnetic moment $\mu_{\text {eff }}=10.6 \mu_{\mathrm{B}} / D y$, which is consistent with previous results [41, 42]. A sharp peak develops at $T_{N}=10 \mathrm{~K}$, signaling the onset of the AFM phase transition, consistent with earlier reports [42-44]. 


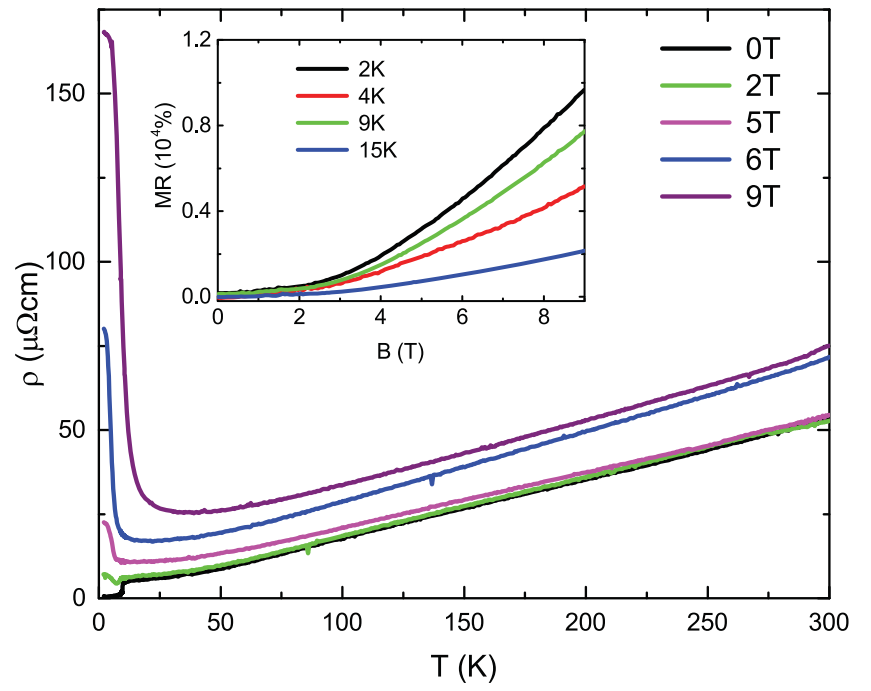

Figure 3. The temperature dependent resistivity $\rho$ of DySb at selected magnetic field.

Figure 3 shows the temperature dependent resistivity $\rho$ of DySb at selected magnetic fields. At zero magnetic field, the resistivity decreases continuously upon cooling until $10 \mathrm{~K}$, then it demonstrates a remarkable drop, which is coincident with the AFM phase transition temperature. Therefore the anomaly feature in resistivity can be ascribed to the magnetic phase transition. In an applied magnetic field, there is a positive magnetoresistivity, which is more significant at low temperatures. At $H=2 \mathrm{~T}$, the resistivity first shows a drop initially and then followed by an increase. For magnetic field $H \geqslant 5 \mathrm{~T}$, the resistivity drop around $10 \mathrm{~K}$ is no longer visible and instead, it increases markedly but starts to saturate below $5 \mathrm{~K}$. This behavior indicates the XMR phenomenon. To further illustrate the XMR phenomenon, we plot magnetoresistance (MR) versus magnetic field $(B)$ at different temperatures for DySb in the inset of figure 3, where MR is defined as $\mathrm{MR}=(\rho(B)-\rho(0)) / \rho(0)$. The MR reaches as large as $1.08 \times 10^{4} \%$ at $2 \mathrm{~K}$ and $9 \mathrm{~T}$, without any sign of saturation, which is consistent with the XMR behavior revealed in [41]. Similar XMR effect is previously reported in AFM semimetal $\mathrm{NdSb}$ [18] and CeSb [20, 22, 25, 26].

Figure 4 shows the reflectance spectra $R(\omega)$ of DySb at selected temperatures in the frequency range from 120 to $8000 \mathrm{~cm}^{-1}$. As seen, $R(\omega)$ at low frequency are rather high, approaching unity at zero frequency limit at all temperatures, and increase with decreasing temperatures. This is a typical metallic response, consistent with the resistivity data at zero magnetic field. With increasing frequency, $R(\omega)$ decreases, reaching a minimum value at about $4000 \mathrm{~cm}^{-1}$ at $300 \mathrm{~K}$, usually referred to the 'screened' plasma edge [39, 45-53]. The relatively low frequency of the 'screened' plasma edge might be ascribed to its semimetal response. As temperatures decreased, the 'screened' plasma edge shifts to higher frequency (i.e. a blueshift), reaching about $4700 \mathrm{~cm}^{-1}$ at $10 \mathrm{~K}$. These results are similar to the reported optical spectroscopy of its isostructural compound LaSb [39].

In contrast to the simple band behavior expected for a low carrier density system like LaSb [39], we find three

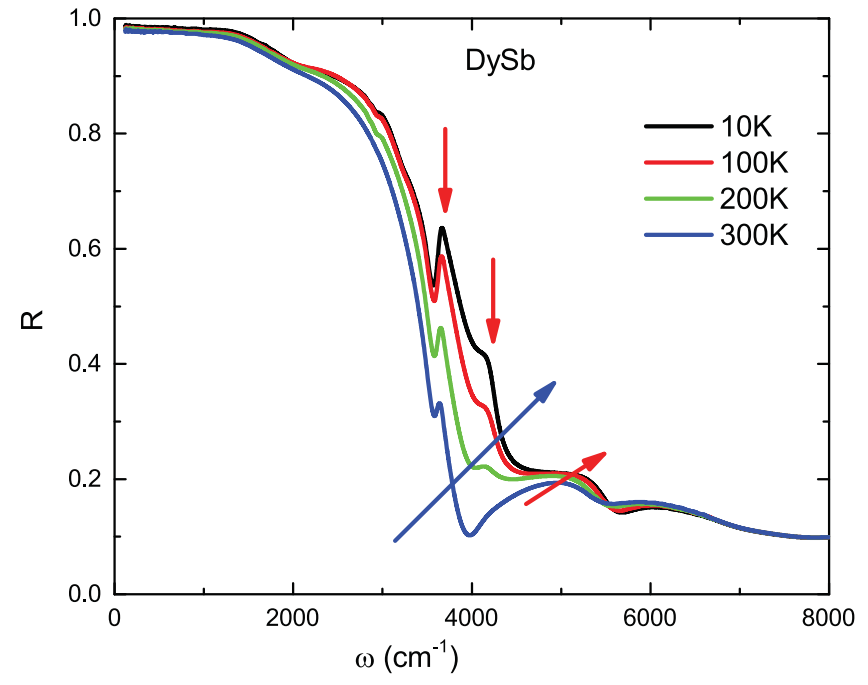

Figure 4. The temperature dependent $R(\omega)$ in the frequency range from 120 to $8000 \mathrm{~cm}^{-1}$. The red arrows indicate the position of the three peak-like midinfrared absorption features. The blue arrow indicates the shift of the 'screened' plasma edge.

peak-like midinfrared absorption features in the vicinity of the 'screened' plasma edge, indicated by red arrows in figure 4 . The first two absorption features occur around the 'screened' plasma edge, which are centered at $3650 \mathrm{~cm}^{-1}$ and $4150 \mathrm{~cm}^{-1}$, respectively. In addition, their central frequency show little shift with temperature. The origin of these two peak-like features might be intraband transition induced by the exchange interaction between the spin of carriers and the localized magnetic moment at each site of rare-earth ion [38]. However, the third absorption feature occurs at higher energies than the 'screened' plasma edge, which centered around $5200 \mathrm{~cm}^{-1}$ appears to move to higher energies as the temperature is lowered. The absorption feature at such a high energy is usually be ascribed to the interband transitions. However, one would not expect a prominent temperature dependence for an interband transition. So the temperature-dependent peak-like feature must have a different origin.

In order to get further information about the temperaturedependent peak-like feature around the 'screened' plasma edge, we plot the real parts of the dielectric function $\epsilon_{1}(\omega)$ as a function of frequency at different temperature, as shown in figure 5. In $\epsilon_{1}(\omega)$, the zero-crossing frequency corresponding to the 'screened' plasma edge in the reflectance spectrum, represents the 'screened' plasma frequency $\omega_{P}^{*}$. As temperature decreases, the 'screened' plasma frequency increases (figure $6(a))$, which is in good agreement with the reflectance plasma minimum. The inflection point $\omega_{\tau}$ in $\epsilon_{1}(\omega)$ at about $5200 \mathrm{~cm}^{-1}$ corresponding to the third absorption feature in $R(\omega)$, (shown as the red arrows in the inset to figure 5) also increases as temperature decreasing. This inflection behavior may anticipate a second zero crossing of ' 1 ', which could result in a characteristic 'second plasma edge'.

The temperature dependent inflection point $\omega_{\tau}$ in $\epsilon_{1}(\omega)$ is reminiscent of the temperature dependent 'screened' plasma edge. In fact, it almost exactly tracks the temperature dependence of the 'screened' plasma frequency $\omega_{P}^{*}$, as shown in 


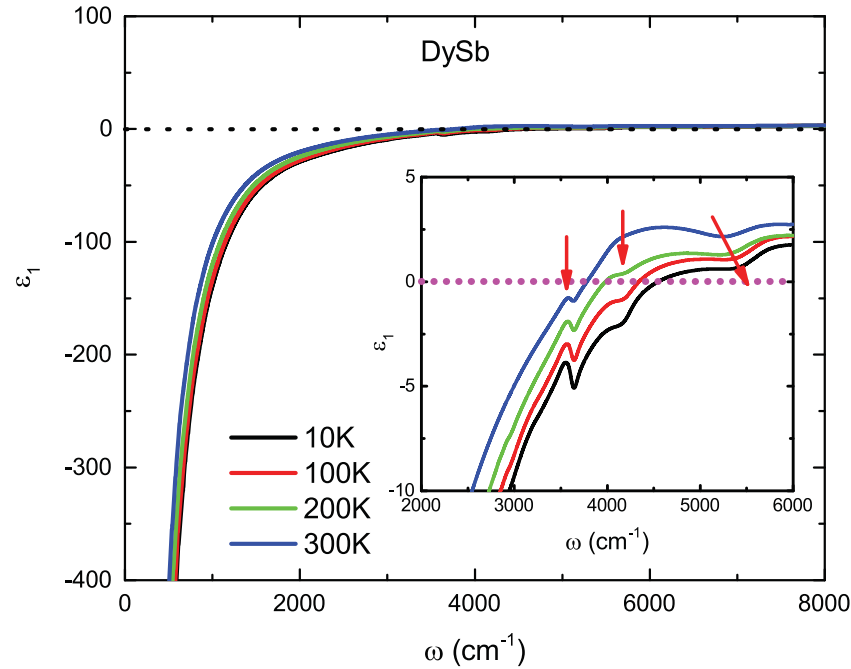

Figure 5. The real part of the dielectric functions $\epsilon_{1}(\omega)$ of DySb in the frequency range from 0 to $8000 \mathrm{~cm}^{-1}$. The red arrows indicate the position of the three peak-like midinfrared absorption features. Inset: the expanded plot of $\epsilon_{1}(\omega)$ from 2000 to $6000 \mathrm{~cm}^{-1}$.

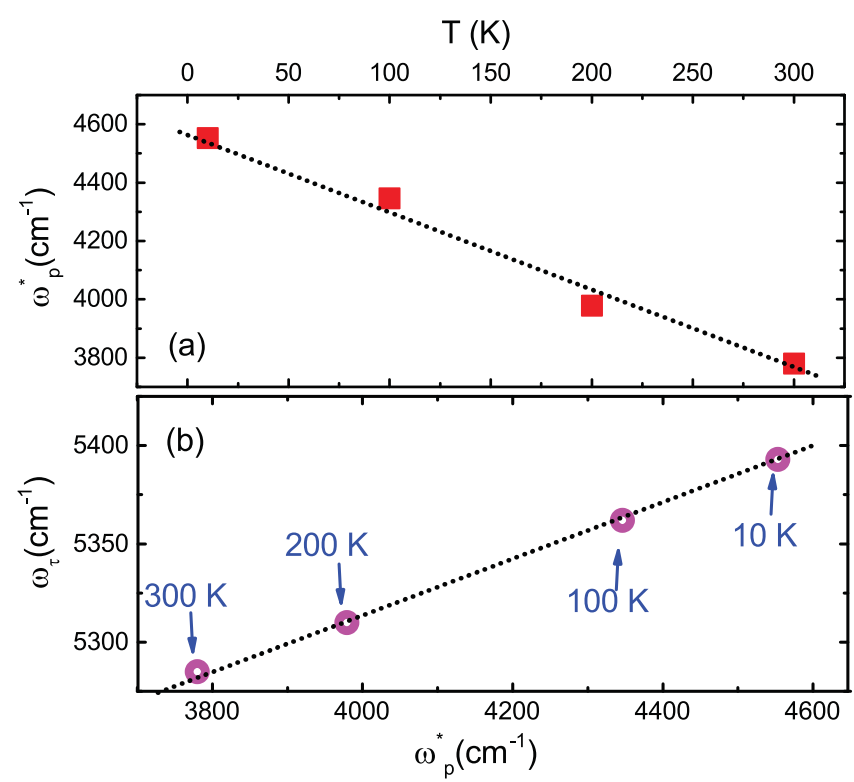

Figure 6. (a) The 'screened' plasma frequency obtained from the peak position of $\operatorname{Im}[-1 / \epsilon(\omega)]$ of DySb. (b) A parametric plot $\omega_{\tau}$ versus $\omega_{P}^{*}$ obtained from the real parts of the dielectric function $\epsilon_{1}(\omega)$

figure 6(b), where we plot the frequency of the inflection point $\omega_{\tau}$ versus $\omega_{P}^{*}$. Based on the figure $6(\mathrm{~b})$, we can identify that $\omega_{\tau}$ show almost a linear dependence of $\omega_{P}^{*}$.

These special phenomena around the 'screened' plasma edge, might be ascribed to the appearance of coupled electron-plasmon 'plasmaron' feature. This is an electron-boson interaction similar to that considered in the context of electron-phonon or electron-magnon scattering. Such an interaction has been anticipated by Lundqvist [54, 55], and observed in quasi-freestanding graphene by angle-resolved photoemission spectroscopy (ARPES) [56], what's more, intrinsic 'plasmaron' also appear in warm graphene [57]. The 'plasmaron' feature in quasi-freestanding graphene and warm graphene share some similarities, such as: in the larger $x=q / T$ region, the 'plasmaron' energy is always below the fermion energy; and the 'plasmaron' and fermion are the same at $q=0$. However, they also have many sharp differences, thermal mass, fermion channel, 'plasmaron' channel and the dispersion of 'plasmaron' et al.

Optically excited 'plasmaron' feature have rarely been observed, which makes the observation particularly interesting. In the case of elemental bismuth, a 'plasmaron' excitation is observed at a higher energy than the plasma edge [46, 47]. For $\mathrm{Na}_{3} \mathrm{Bi}$ the 'plasmaron' excitation is observed below the the plasma edge [58]. DySb is another example besides bismuth and $\mathrm{Na}_{3} \mathrm{Bi}$, which show clear optical evidence for a 'plasmaron' feature. Bismuth, $\mathrm{Na}_{3} \mathrm{Bi}$ and $\mathrm{DySb}$ have many similarities, such as small FS, low carrier density and high Fermi velocity. These observations suggest that the "plasmaron' feature are perhaps more ubiquitous, and open up the possibility of further investigation for such collective modes in the various types of low carrier density systems.

However, the 'plasmaron' feature is not observed in $\mathrm{LaSb}$ [39], the isostructural compound of DySb. Note that Dy has $4 f$ electrons while $f$ electrons is absent in La. The different $4 f$ occupation may give rise to different magnetic properties and electronic structures. In addition, DySb is a typical Ising antiferromagnet [2, 42, 43, 59-62], while LaSb is an electronhole compensated semimetals $[14,16]$. The difference in $4 f$ electron occupation and ground state may have different influence on the band structure of the two compounds, which could be the reason for the different features in the vicinity of the 'screened' plasma edge.

Nevertheless, the 'screened' plasma frequency $\omega_{p}^{*}$ of DySb increases with decreasing temperature, similar to LaSb [39]. We know that the 'screened' plasma frequency $\omega_{p}^{*}$ is linked to the plasma frequency $\omega_{p}$ by the relation $\omega_{p}^{*}=\omega_{p} / \sqrt{\varepsilon_{\infty}}$. The plasma frequency satisfies the equation $\omega_{P}^{2}=4 \pi n e^{2} / \mathrm{m}^{*}$, where $\mathrm{n}$ is the carrier density, $m^{*}$ is the effective mass, and $\varepsilon_{\infty}$ is the dielectric constant at high frequency. So the increase in the 'screened' plasma frequency indicates an increase of $n / m^{*}$. It is reported in HoSb that the carrier density decreases as the temperature decreases from 300 to $10 \mathrm{~K}$ [63]. Since DySb is an isostructural compound of HoSb, the carrier density of the former could have similar temperature dependence as that of latter. Therefore, the fact that the 'screened' plasma frequency of DySb increases with decreasing temperature could be ascribed to the reduction of effective mass $m^{*}$. Furthermore, the reduction of effective mass $m^{*}$ can be explained by the change of dispersion near $E_{\mathrm{F}}$ and a 'three-band' model that is at high temperatures heavier states lying close to $E_{\mathrm{F}}$ become thermally populated leading to a reduction in $\omega_{P}^{*}$, like the explanation in $\mathrm{LaSb}$ [39].

\section{Conclusion}

In summary, we report magnetic susceptibility, resistivity and optical spectroscopy study on single crystal sample DySb. It exhibits an AFM phase transition at $T_{N}=10 \mathrm{~K}$ and XMR is observed at low temperatures. Optical measurements indicate that the material has a low carrier density and the screened 
plasma edge increases with decreasing temperature. Most remarkably, our study reveals several special features in the vicinity of the screened plasmon frequency, which could be due to the appearance of the coupled electron-plasmon 'plasmaron'.

\section{Acknowledgments}

The work at HPSTAR is supported by NSAF, Grant No. U1530402. The work at IOP is supported by the National Basic Research Program of China (Grant No. 2014CB921504), the National Science Foundation of China (Grant No.11634015).

\section{ORCID iDs}

\section{H Xiao 이 https://orcid.org/0000-0001-8859-9967}

\section{References}

[1] Busch G and Vogt O 1968 J. Appl. Phys. 391334

[2] Everett G E and Streit P 1979 J. Magn. Magn. Mater. 12277

[3] Shirotani I, Hayashi J, Yamanashi K, Ishimatsu N, Shimomura O and Kikegawa T 2001 Phys. Rev. B 64132101

[4] Mullen M E, Lüthi B, Wang P S, Bucher E, Longinotti L D, Maita J P and Ott H R 1974 Phys. Rev. B 10186

[5] Gupta D C and Kulshrestha S 2011 J. Alloys Compd. 5094653

[6] Pagare G, Soni P, Srivastava V and Sanyal S P 2009 J. Phys. Chem. Solids 70650

[7] Cui S, Feng W, Hu H, Feng Z and Liu H 2009 Solid State Commun. 149996

[8] De M and De S 1999 J. Phys. Chem. Solids 60337

[9] Pagare G, Sen D, Srivastava V and Sanyal S P 2010 J. Phys.: Conf. Ser. 215012114

[10] Taub H and Parente C 1975 Solid State Commun. 16857

[11] Vogt O and Mattenberger K 1993 Lanthanides/Actinides: Physics I (Handbook on the Physics and Chemistry of Rare Earths vol 17) (Amsterdam: Elsevier) pp 301-407

[12] Rossat-Mignod J, Burlet P, Villain J, Bartholin H, Tcheng-Si W, Florence D and Vogt O 1977 Phys. Rev. B 16440

[13] Cooper B R and Vogt O 1970 Phys. Rev. B 11211

[14] Zeng L K et al 2016 Phys. Rev. Lett. 117127204

[15] Niu X H, Xu D F, Bai Y H, Song Q, Shen X P, Xie B P, Sun Z, Huang Y B, Peets D C and Feng D L 2016 Phys. Rev. B 94165163

[16] Guo P J, Yang H C, Zhang B J, Liu K and Lu Z Y 2016 Phys. Rev. B 93235142

[17] Tafti F F, Gibson Q D, Kushwaha S K, Haldolaarachchige N and Cava R J 2015 Nat. Phys. 12272

[18] Wakeham N, Bauer E D, Neupane M and Ronning F 2016 Phys. Rev. B 93205152

[19] Wu F, Guo C Y, Smidman M, Zhang J L and Yuan H Q 2017 Phys. Rev. B 96125122

[20] Wu Y, Lee Y, Kong T, Mou D, Jiang R, Huang L, Bud'ko S L, Canfield P C and Kaminski A 2017 Phys. Rev. B 96035134

[21] Ye L, Suzuki T, Wicker C R and Checkelsky J G 2018 Phys. Rev. B 97081108

[22] Guo C, Cao C, Smidman M, Wu F, Zhang Y, Steglich F, Zhang F C and Yuan H 2017 NPJ Quantum Mater. 239

[23] Takayama A, Souma S, Sato T, Arakane T and Takahashi T 2009 J. Phys. Soc. Japan 78073702

[24] Zeng M, Fang C, Chang G, Chen Y A, Hsieh T, Bansil A, Lin H and Fu L 2016 (arXiv:1504.03492)

[25] Oinuma H et al 2017 Phys. Rev. B 96041120
[26] Alidoust N et al 2016 (arXiv:1604.08571)

[27] Kasuya T, Sera M, Okayama Y and Haga Y 1996 J. Phys. Soc. Japan 65160

[28] Duan C G, Sabiryanov I F, Mei W N, Dowben P A, Jaswal S and Tsymbal E Y 2007 J. Phys.: Condens. Matter 19315220

[29] Pavlosiuk O et al 2017 Sci. Rep. 712822

[30] Ali M N et al 2014 Nature 514205

[31] Feng J, Pang Y, Wu D, Wang Z, Weng H, Li J, Dai X, Fang Z, Shi Y and Lu L 2015 Phys. Rev. B 92081306

[32] Liang T, Gibson Q, Ali M N, Liu M, Cava R J and Ong N P 2014 Nat. Mater. 14280

[33] Huang X et al 2015 Phys. Rev. X 5031023

[34] Shekhar C et al 2015 Nat. Phys. 11645

[35] Kwon Y, Takeshige M, Nakamura O, Suzuki T and Kasuya T 1991 Physica B 171316

[36] Kimura S, Kitazawa H, Kido G and Suzuki T 2000 J. Phys. Soc. Japan 69647

[37] Kwon Y, Takeshige M, Suzuki T and Kasuya T 1990 Physica B 163328

[38] Kwon Y, Jung M, Lee K, Kimura S and Suzuki T 1997 Physica B 24088

[39] Ban W J, Guo W T, Luo J L and Wang N L 2017 Chin. Phys. Lett. 34077804

[40] Tanner D B 2015 Phys. Rev. B 91035123

[41] Liang D D, Wang Y J, Xi C Y, Zhen W L, Yang J, Pi L, Zhu W K and Zhang C J 2018 APL Mater. 6086105

[42] Li D, Nimori S and Shikama T 2010 Solid State Commun. 1501865

[43] Hu W J, Du J, Li B, Zhang Q and Zhang Z D 2008 Appl. Phys. Lett. 92192505

[44] Bucher E, Birgeneau R J, Maita J P, Felcher G P and Brun T O 1972 Phys. Rev. Lett. 28746

[45] Dordevic S V, Wolf M S, Stojilovic N, Nikolic M V, Vujatovic S S, Nikolic P M and Tung L C 2012 Phys. Rev. B 86115119

[46] Armitage N P, Tediosi R, Lévy F, Giannini E, Forro L and van der Marel D 2010 Phys. Rev. Lett. 104237401

[47] Tediosi R, Armitage N P, Giannini E and van der Marel D 2007 Phys. Rev. Lett. 99016406

[48] Dordevic S V, Foster G M, Wolf M S, Stojilovic N, Lei H, Petrovic C, Chen Z, Li Z Q and Tung L C 2016 J. Phys.: Condens. Matter 28165602

[49] Li G, Hu W Z, Qian D, Hsieh D, Hasan M Z, Morosan E, Cava R J and Wang N L 2007 Phys. Rev. Lett. 99027404

[50] Caimi G, Perucchi A, Degiorgi L, Ott H R, Pereira V M, Neto A H C, Bianchi A D and Fisk Z 2006 Phys. Rev. Lett. 96016403

[51] Li G, Hu W Z, Dong J, Qian D, Hsieh D, Hasan M Z, Morosan E, Cava R J and Wang N L 2007 Phys. Rev. Lett. 99167002

[52] Dordevic S V, Wolf M S, Stojilovic N, Lei H and Petrovic C 2013 J. Phys.: Condens. Matter 25075501

[53] Dong T, Yuan R-H, Shi Y-G and Wang N-L 2013 Chin. Phys. Lett. 30127801

[54] Lundqvist B I 2010 Phys. Kondens. Mater. 6193

[55] Lundqvist B I 2010 Physica Status Solidi 32273

[56] Bostwick A, Speck F, Seyller T, Horn K, Polini M, Asgari R, Macdonald A H and Rotenberg E 2010 Science 328999

[57] Liu D, Chen S, Zhang S and Ma N 2017 J. Phys.: Condens. Matter 29425601

[58] Jenkins G S et al 2016 Phys. Rev. B 94085121

[59] Child H R, Wilkinson M K, Cable J W, Koehler W C and Wollan E O 1963 Phys. Rev. 131922

[60] Busch G, Marinek O, Menth A and Vogt O 1965 Phys. Lett. 14262

[61] Felcher G P, Brum T O, Gambino R J and Kuznietz M 1973 Phys. Rev. B 8260

[62] Levy P M and Chen H H 1971 Phys. Rev. Lett. 271385

[63] Wang Y Y, Sun L L, Xu S, Su Y and Xia T L 2018 Phys. Rev. B 98045137 